\title{
EXCITATION WAVELENGTH AND VISCOSITY DEPENDENCE OF LANDAU-ZENER ELECTRONIC TRANSITIONS IN CONDENSED MEDIA
}

\author{
Biman BAGCHI \\ Solid State and Structural Chemistry Unit, Indian Institute of Science, Bangalore 560012, India
}

Received 7 February 1986; in final form 13 May 1986

\begin{abstract}
We investigate the excitation wavelength and solvent viscosity dependence of the rate of formation and distribution of ground-state isomers in a Landau-Zener (LZ) electronic transition from the excited state. We find that both the rate and the distribution depend strongly on the excitation wavelength. A viscosity-mediated cross-over from a dynamic LZ transition to one governed by an exponential gap law is predicted.
\end{abstract}

\section{Introduction}

Avoided crossings of potential surfaces play an important role in the interpretation of organic photochemical reactions [1-3]. An essential ingredient for our understanding of these processes is the expression for the transition probability derived many years ago by Landau and Zener $[4,5]$. This expression is usually given in the following form

$$
p=\exp \left(-\gamma(\Delta E)^{2} / v\right)
$$

where $\Delta E$ is the energy gap between the two adiabatic surfaces at the crossing point of the associated diabatic surfaces, $v$ is the velocity with which the system passes through the crossing region. $\gamma$ is given by [5]

$$
\gamma=(2 \pi)^{2} / h\left(F_{2}-F_{1}\right)
$$

where $F_{1}, F_{2}$ are the "forces" acting on the two crossing diabatic surfaces and $h$ is Planck's constant.

In this Letter, we are interested in the consequences of avoided crossings on photochemical reactions in condensed media. Consequently we need to generalize eq. (1). One effect of the interaction of the reactive system with the solvent molecules is to broaden the Landau-Zener transition zone. In this case we have to consider a position-dependent transition probability $p(x)$ which will be sharply peaked in the region of avoided crossing. At this juncture we would like to point out that if electronic relaxation is much faster than solvent relaxation (that is, in the limit of static modulation), the broadening of the energy surfaces may be of inhomogeneous character. In the opposite limit of faster solvent relaxation, the broadening may be homogeneous. Experimental situations [1-7] may vary widely between these two limits and it may be necessary to deal with each specific case separately for quantitative purposes. Here we assume that the decay from the excited surface can be described by a position-dependent sink whose functional form we assume to be still given by eq. (1), with $\Delta E$ and $v$ now position dependent. The details of the solute--solvent interactions may be parameterized by $\Delta E$ and $v$. The assumption of a positiondependent sink is useful, as shown below, in obtaining general, qualitative results. For a general potential surface, $F_{2}-F_{1}$ may also depend on the position coordinate, but this position dependence is negligible for the harmonic surfaces that we assume in this work.

* Contribution number 354 from Solid State and Structural Chemistry Unit. 
Let us assume that the ground state has two isomeric forms, $A$ and $B$. Let the molecule be excited by a light pulse of suitable wavelength from the well $A$ and placed at time $t=0$ at the position $x=x_{0}$ on the excited state surface. Here $x$ denotes the reaction coordinate. Subsequent relaxation of the system will bring it down towards the avoided-crossing region where the transition probability $p$, given by eq. (1), is large. If $p$ is close to unity, then most of the product will be formed in well B. On the other hand, if $p$ is very small $(p \ll 1)$ in the avoided-crossing region, then the ground state isomers $A$ and $B$ will be formed in almost equal amounts. The situation, however, is not so clear when $p$ takes intermediate values. Another interesting point in LZ transitions is that the product distribution may depend on the excitation wavelength. The rate of formation of the ground-state products may also depend on the excitation wavelength. For isomerization reactions the solvent viscosity may also have an important effect both on the rate of formation and on the distribution of ground-state isomers. We are not aware of any detailed calculation of these quantities and their interdependences.

In this Letter we present simple analytic expressions for the following quantities: (a) the distribution of groundstate products formed by $\mathrm{LZ}$ transitions from the initially formed excited state, (b) the excitation wavelength dependence of the rate of product formation and (c) the effects of viscosity on the rate of formation and on the distribution of ground-state products. We believe that these expressions are new and will be useful in understanding organic photochemical reactions. A detailed presentation of the results along with comparison with existing experimental data will be published elsewhere [8].

\section{Distribution of ground-state isomers}

In this section we present expressions for the fraction of products, $F_{\mathrm{A}}$ and $F_{\mathrm{B}}$, formed by $\mathrm{LZ}$ transitions from the excited state. These expressions are valid in the limit of small solute-solvent interactions; we shall consider medium effects later.

Subsequent to the initial excitation, the system relaxes towards the avoided-crossing region. There is a finite probability of decay as the system passes through the avoided-crossing region. Let $f_{\mathrm{B}}$ denote the probability of transition to well B when the system passes from left to right and $f_{\mathrm{A}}$ denote the transition probability in the reverse situation. Note that $f_{\mathrm{A}}$ and $f_{\mathrm{B}}$ are different from the Landau-Zener transition probability $p$ given by eq. (1). Here they represent the total transition probability on each passage across the avoided crossing region. They may be obtained by integrating eq. (1) over a trajectory. We shall evaluate $f_{\mathrm{A}}$ and $f_{\mathrm{B}}$ in section 3 . Here we just make the observation that they will depend both on the ground and the excited state potential surfaces. In general $f_{\mathrm{A}}$ and $f_{\mathrm{B}}$ will be unequal. Let $F_{\mathrm{A}}$ and $F_{\mathrm{B}}$ denote the fraction of isomers formed in the wells $\mathrm{A}$ and $\mathrm{B}$, respectively. Then our scheme is as follows. In the absence of interactions with other degrees of freedom, the system oscillates back and forth on the excited surface. We can calculate the transition probability on each passage across the avoided crossing region and sum up all the contributions to obtain $F_{\mathrm{A}}$ and $F_{\mathrm{B}}$. Then $F_{\mathrm{A}}$ is given by

$$
F_{\mathrm{A}}=f_{\mathrm{A}}\left(1-f_{\mathrm{B}}\right)+f_{\mathrm{A}}\left(1-f_{\mathrm{B}}\right)^{2}\left(1-f_{\mathrm{A}}\right)+f_{\mathrm{A}}\left(1-f_{\mathrm{B}}\right)^{3}\left(1-f_{\mathrm{A}}\right)^{2}+\ldots=f_{\mathrm{A}}\left(1-f_{\mathrm{B}}\right) /\left[1-\left(1-f_{\mathrm{B}}\right)\left(1-f_{\mathrm{A}}\right)\right] .
$$

Similarly, $F_{\mathrm{B}}$ is given by

$$
F_{\mathrm{B}}=f_{\mathrm{B}}+f_{\mathrm{B}}\left(1-f_{\mathrm{B}}\right)\left(1-f_{\mathrm{A}}\right)+f_{\mathrm{B}}\left(1-f_{\mathrm{B}}\right)^{2}\left(1-f_{\mathrm{A}}\right)^{2}+\ldots=f_{\mathrm{B}} /\left[1-\left(1-f_{\mathrm{B}}\right)\left(1-f_{\mathrm{A}}\right)\right] \text {. }
$$

In summing the above series, we used $f_{\mathrm{A}}, f_{\mathrm{B}}>0$. The series does not converge for $f_{\mathrm{B}}=0$ which is unphysical. Note that $F_{\mathrm{A}}+F_{\mathrm{B}}=1$.

Several comments on eqs. (2) and (3) are in order. Firstly, $F_{\mathrm{B}}$ is always greater than $F_{\mathrm{A}}$ for $f_{\mathrm{B}}=f_{\mathrm{A}}=f$, for non-zero values of $f$. As $f \rightarrow 0, F_{\mathrm{B}} \rightarrow F_{\mathrm{A}}$ in a non-linear fashion depicted in fig. 1. $F_{\mathrm{B}}$ rises slowly at first as $f$ is increased from zero, rising more rapidly as $f \rightarrow 1$. In the limit $f \rightarrow 1, f_{\mathrm{B}}$ is linearly dependent on $f, F_{\mathrm{B}} \propto f$. Secondly, eqs. (2) and (3) predict that even for a fairly large value of $f$, approximately equal amounts of $\mathrm{A}$ and $\mathrm{B}$ will form.

Some insight into the behaviour for the general case $f_{\mathrm{A}} \neq f_{\mathrm{B}}$ can be obtained by considering a small asymme- 


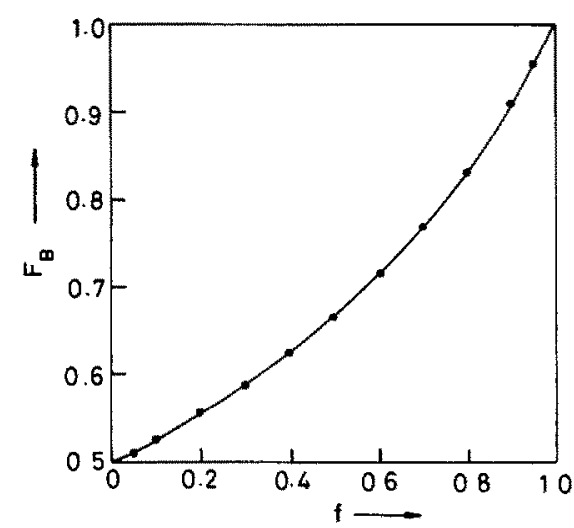

Fig. 1. The fraction of $B$ isomer, $F_{B}$, plotted against the function $f$.

try. Let $f_{\mathrm{A}}=f$ and $f_{\mathrm{B}}=f+\delta$ with $\delta$ small. Then a first-order correction to $F_{\mathrm{B}}$ is given by

$$
F_{\mathrm{B}}=F_{\mathrm{B}}^{0}\left\{1+\delta\left[1-(1-f)^{2}\right]\right\} \text {, }
$$

where $F_{B}^{0}$ is the fraction of $B$ for the symmetric case $(\delta=0)$. Eq. (4) predicts that the effect of the asymmetry depends on the value of $f$ itself, being larger for small $f$ and vice versa. This is expected.

\section{Excitation wavelength dependence of the rate of formation and distribution of ground-state products.}

In this section we shall present an expression for the rate of formation of ground-state isomers formed by LZ transitions from the initially prepared excited state. The expression is derived under the assumptions that the excited potential surface can be approximated by a harmonic well of frequency $\omega$ centred around the minimum on the surface and that interactions with non-reactive degrees of freedom are negligible. We shall also present an expression for the factor $f$ - the decay from the excited-state surface on each passage through the avoided crossing region.

We first note that a rate-law behaviour can be expected only for small values of the total transition probability $f$. Also, in this limit, the rates of formation of the two isomers are approximately equal. So, it suffices to calculate the rate of decay of population from the excited surface. Secondly, the $L Z$ transition probability $p$ depends on the position coordinate $x$ through its dependence on the energy gap $\Delta E$ and the velocity $v$. It is, therefore, convenient to write the transition probability in the following form

$$
p(x)=p_{0}\left(v_{0}\right) S\left(x \mid x_{0}, v_{0}\right),
$$

where $p_{0}$ is the probability of decay from the minimum on the potential surface (where the probability of decay is a maximum) and $S(x)$ is a sink function that contains the position dependence of the transition probability. Here $x_{0}=x(t=0)$ and $v_{0}=v(t=0)$. The forms of $p_{0}$ and $S(x)$ can be obtained from eq. (1).

-Next we estimate the change in population density after each passage through the sink region. We follow the procedure outlined in refs. [9-12]. Let us consider a solute particle, initially at the phase point $\left(x_{0}, v_{0}\right)$, undergoing inertial motion in a harmonic well of frequency $\omega$. The amount of time spent by the particle in a region between $x$ and $x+\mathrm{d} x$ is

$$
\mathrm{d} x /\left[v_{0}^{2}+\omega^{2}\left(x_{0}^{2}-x^{2}\right)\right]^{1 / 2} .
$$

The probability of absorption by the sink at each $x$ is $p_{0}(x) S(x)$ and, therefore, the total absorption probability per particle over a short time is 


$$
p_{0}\left(v_{0}\right) \int_{x\left(t \mid x_{0}, v_{0}\right)} \mathrm{d} x\left[v_{0}^{2}+\omega^{2}\left(x_{0}^{2}-x^{2}\right)\right]^{-1 / 2} S\left(x \mid x_{0}, v_{0}\right)
$$

The above expression is a line integral over the particle trajectory, $x\left(t \mid x_{0}, v_{0}\right)$. If we sample from a thermal distribution for $v_{0}$, then the fractional change in excited-state population during a single passage through the avoided crossing region is given by

$$
f_{\mathrm{e}}=\frac{\int_{-\infty}^{\infty} \mathrm{d} v_{0} p_{0}\left(v_{0}\right) \exp \left(-\beta \mu v_{0}^{2}\right) \int_{x\left(t \mid x_{0}, v_{0}\right)} \mathrm{d} x\left[v_{0}^{2}+\omega^{2}\left(x_{0}^{2}-x^{2}\right)\right]^{-1 / 2} S\left(x \mid x_{0}, v_{0}\right)}{\int_{-\infty}^{\infty} \mathrm{d} v_{0} \exp \left(-\beta \mu v_{0}^{2}\right)},
$$

where $\mu$ is the effective mass of the reactive motion, $T$ is the temperature and $\beta=\left(k_{\mathrm{B}} T\right)^{-1}$, with $k_{\mathrm{B}}$ the Boltzmann constant.

Before we calculate $f_{\mathrm{e}}$, let us relate the rate of decay to $f_{\mathrm{e}}$. Since the excited-state population can change only during the particle's passage through the sink, the total population, $P_{e}$, remaining on the excited state surface after $n$ passes is

$$
P_{\mathrm{e}}(t=n \pi / \omega)=\left(1-f_{\mathrm{e}}\right)^{n} P_{\mathrm{e}}(0),
$$

and, therefore

$$
\ln P_{\mathrm{e}}(t=n \pi / \omega)=n \ln \left(1-f_{\mathrm{e}}\right)+\ln P_{\mathrm{e}}(0)
$$

From (10), we can derive an expression for the "coarse-grained" rate constant $k$ [9]

$$
k=-(\omega / \pi) \ln \left(1-f_{\mathrm{e}}\right) \approx \omega f_{\mathrm{e}} / \pi, \quad f_{\mathrm{e}} \ll 1 .
$$

The last approximation is consistent with our assumption that the change in total population during a single pass through the sink region is small. In the other limit, no rate constant can exist. Next, we evaluate $f_{\mathrm{e}}$ by evaluating the integral in eq. (8).

We first note that the sink function is sharply peaked near the origin. The potential energy of the solute particle in the sink region is then negligible compared to the initial kinetic energy of the particle, so that $v_{0}^{2}+\omega^{2} x_{0}^{2}$ $\gg \omega^{2} x^{2}$ near $x \approx 0$. Then $\omega^{2} x^{2}$ can be omitted from eq. (8). We further assume $\omega^{2} x_{0}^{2}>k_{\mathrm{B}} T / \mu$. The last assumption is expected to be valid for some, if not for all, isomerization reactions [10]. With these assumptions, $f_{\mathrm{e}}$ can be evaluated analytically to obtain

$$
f_{\mathrm{e}}=\left[\gamma \mu\left(\Delta E_{0}\right)\left(\omega+\omega_{\mathrm{b}}\right) / \omega x_{0}\right]^{-1 / 2} \exp \left[-\gamma\left(\Delta E_{0}\right)^{2} / 2 \omega x_{0}\right] I\left(\omega x_{0} / 4 \gamma\left(\Delta E_{0}\right)^{2}\right)
$$

where

$$
I(\nu)=\int_{-\infty}^{\infty} \mathrm{d} x \exp \left(-x^{2}-\nu x^{4}\right)
$$

$\Delta E_{0}$ is the energy difference between the ground and the excited surfaces at $x=0$ (see fig. 1) and $\omega_{\mathrm{b}}$ is the harmonic frequency that characterizes the barrier region of the ground-state surface.

Several comments on the expression (12) are in order. Firstly, it has the expected dependence of rate on $\left(\Delta E_{0}\right)^{2}$. The prefactor of this term in the exponential is new. Secondly, the expression shows a dependence of the rate on the frequency $\omega_{b}$ of the ground state barrier; however, this dependence is rather weak, $\propto\left(\omega+\omega_{b}\right)^{-1 / 2}$. Thirdly, and most importantly, $f_{\mathrm{e}}$ - and hence the rate $k$ - shows a strong dependence on $x_{0}$, the initial position on the excited surface.

As discussed earlier, for small value of $f, f_{\mathrm{e}}$ is identical with $f\left(=f_{\mathrm{A}}=f_{\mathrm{B}}\right)$ of eqs. (2) and (3). Thus, eq. (12) predicts a strong dependence of the product distribution terms $F_{\mathrm{A}}$ and $F_{\mathrm{B}}$ on $x_{0}$. 


\section{Medium effects}

In this section, we present an expression for the medium effects on the LZ transition probability $p$, and hence on $f$ and $k$. The original derivation of the $\mathrm{LZ}$ formula was based on a semiclassical formalism. It is, of course, desirable to modify that treatment to include the medium effects. We are aware of no calculation which is appropriate for the present avoided-crossing problem. In this work, we have adopted a simpler, somewhat ad hoc, approach. We shall consider that the reactive motion is coupled to external degrees of freedom and that the equation of motion of the reactive mode (motion along the coordinate $x$ ) can be described by a Langevin equation in a potential surface with a delta-correlated random force related to the friction parameter $\zeta$ by the first fluctuation-dissipation theorem. The average transition probability $\langle p\rangle$ is then obtained by performing an average over eq. (1),

$$
\langle p\rangle=\left\langle\exp \left(-\gamma(\Delta E)^{2} / v\right)\right\rangle
$$

The main effect of the random forces is to damp the velocity $v$. We expect that the average velocity, hence the average transition probability $\langle p\rangle$, will go to zero rapidly as the value of the friction parameter (which is the measure of solute-solvent coupling) is increased. Here we obtain an analytic expression for the dependence of $\langle p\rangle$ on $\zeta$.

In order to perform the averaging in eq. (14), we proceed in the following manner. First we perform a Laplace transform on eq. (14) to obtain

$$
\langle p(s)\rangle=\left\langle\int_{0}^{\infty} \mathrm{d} X \exp ^{-X s} p(X, v)\right\rangle=\langle v /(1+s v)\rangle,
$$

where $X=\gamma(\Delta E)^{2}$. Next we use the identity

$$
v /(1+s v)=\langle v\rangle /(1+s\langle v\rangle)-(\langle v\rangle-v) /\left[1+s(v+\langle v\rangle)+s^{2} v\langle v\rangle\right],
$$

and make the approximations $¥$

$$
v\langle v\rangle \approx\langle v\rangle^{2}, \quad v+\langle v\rangle \approx 2\langle v\rangle,
$$

to obtain

$$
\langle v /(1+s v)\rangle=\langle v\rangle /(1+s\langle v\rangle)+\left[\langle v\rangle /(1+s\langle v\rangle)^{2}\right]\left\{1-\exp \left[-\left(1 / 2\langle v\rangle^{2}\right)\left(\left\langle v^{2}\right\rangle-\langle v\rangle^{2}\right)\right]\right\} .
$$

Next we substitute (18) in eq. (15) and Laplace invert to get

$$
\langle p(\Delta E)\rangle=\exp \left(-\gamma(\Delta E)^{2} /\langle v\rangle\right)\left\{1+\left[\gamma(\Delta E)^{2} /\langle v\rangle\right]\left\{1-\exp \left[-\left(1 / 2\langle v\rangle^{2}\right)\left(\left\langle v^{2}\right\rangle-\langle v\rangle^{2}\right)\right]\right\}\right\} .
$$

For Brownian motion in a harmonic well, $\langle v\rangle$ and $\left\langle v^{2}\right\rangle$ are given by [13]

$$
\begin{aligned}
& \langle v\rangle=-\left(2 x_{0} \omega^{2} / \beta_{1}\right) \sinh \left(\frac{1}{2} \beta_{1} t\right) \exp \left(-\frac{1}{2} \zeta t\right), \\
& \left\langle v^{2}\right\rangle=k_{\mathrm{B}} T / \mu+\left(4 \omega / \beta_{1}^{2}\right)\left(x_{0}^{2}-k_{\mathrm{B}} T / \mu \omega^{2}\right) \sinh ^{2}\left(\frac{1}{2} \beta_{1} t\right) \exp \left(-\frac{1}{2} \zeta t\right),
\end{aligned}
$$

with

$$
\beta_{1}=\left(\zeta^{2}-4 \omega^{2}\right)^{1 / 2}
$$

We need the average quantities at the times of the solute particle's passage through the avoided crossing region. This can be obtained from the zeros of the average position given by [15]

$$
\langle x\rangle=x_{0}\left[\cosh \left(\frac{1}{2} \beta_{1} t\right)+\left(\zeta / \beta_{1}\right) \sinh \left(\frac{1}{2} \beta_{1} t\right)\right] \exp \left(-\frac{1}{2} \zeta t\right) .
$$

\footnotetext{
* For relaxation in a potential well, these approximations are reasonable.
} 


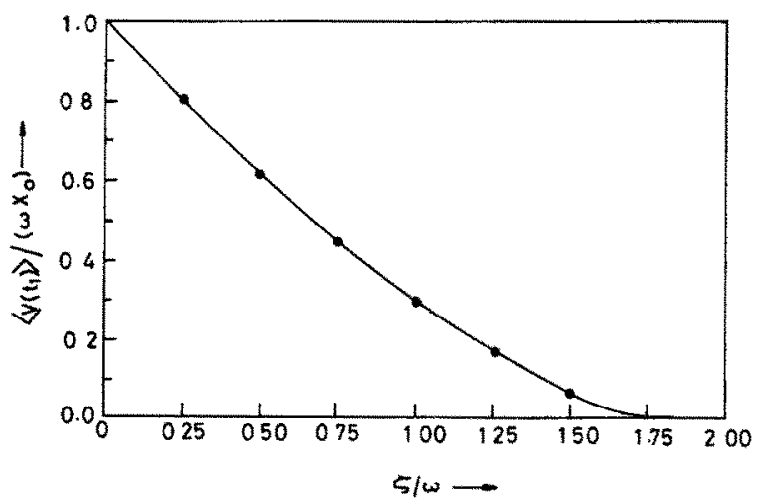

Fig. 2. The average velocity of $\left\langle v\left(t_{1}\right)\right\rangle$ of the system at the time $\left(t_{1}\right)$ of its first passage across the origin $(x=0)$ plotted against the friction parameter $\xi . \omega$ is the frequency of the excited-state potential surface.

In fig. 2, we have plotted the average velocity $\left\langle u\left(t_{1}\right)\right\rangle$, at the time $t_{1}$ of the first passage through $x=0$, as a function of the friction parameter. As can be seen from the figure, $\left\langle u\left(t_{1}\right)\right\rangle$ decreases rapidly as $\zeta$ is increased from zero. This implies that the $\mathrm{LZ}$ transition probability may be severely quenched in the condensed phase if the reactive motion is strongly coupled to the solvent molecules.

The result of this section raises an interesting question: what are the conditions that will give rise to significant LZ transitions? There is obviously a competition between $(\Delta E)^{2}$ and $\langle v\rangle$; both may become small near the avoided crossing region in the condensed phase. The interesting situation is where both are comparable.

In refs. [9-11], an exponential gap law for non-radiative transition probability was assumed in order to describe the electronic relaxation in the absence of an activation barrier. The present case of Landau-Zener transitions is different because this is a dynamic transition that can occur only when the system approaches the avoidedcrossing region with a finite velocity. The exponential gap law has a completely different origin [14]. As the solute-solvent interaction increases, there may be a cross-over from the LZ transition to the one governed by an exponential gap law.

\section{Conclusions}

The main results of this paper are summarized below.

(1) We have obtained an expression (eq. (2)) for the distribution of ground-state isomers formed by LandauZener electronic relaxation of the initially prepared excited state. This result is depicted in fig. 1.

(2) An expression for the rate of formation of the ground state products is obtained (eqs. (9) and (13)). Our result shows a strong dependence on the wavelength of the light used to prepare the excited state. The distribution functions $F_{\mathrm{A}}$ and $F_{\mathrm{B}}$ also depend strongly on excitation wavelength.

(3) We have obtained an approximate expression (eq. (19)) for the dependence of the transition probability on the solvent viscosity. We have argued that LZ transition may be severely quenched in the presence of strong coupling between the reactive mode and other non-reactive modes.

The basis of this paper is purely classical. At low temperatures and in the low-viscosity limit the quantum nature of the vibrational motion will begin to have an effect. However, we do not expect these quantum effects to alter our conclusions significantly.

The theoretical predictions of this paper will be tested against experiment in a separate publication [8]. 


\section{Acknowledgement}

The author is grateful to Professor V. Ramamurthy for interesting discussions and for pointing out useful references. The author thanks Professor C.N.R. Rao, Professor M. Chowdhury, Dr. S. Ramasesha and Dr. P. Ganguly for helpful discussions.

\section{References}

[1] W.G. Dauben, L. Salem and N.J. Turro, Accounts Chem. Res. 8 (1975) 41.

[2] N.J. Turro, Modern molecular photochemistry (Benjamin-Cummings, Menlo Park, 1978).

[3] L. Salem, Israel J. Chem. 14 (1975) 89.

[4] L.D. Landau, Physik. Z. Sowjet 2 (1932) 46.

[5] C. Zener, Proc. Roy. Soc. A137 (1932) 696.

[6] A. Devaquet, Pure Appl. Chem. 41 (1975) 455.

[7] W.G. Dauben, E.L. McInnis and D.M. Michno, in: Rearrangements in ground and excited states, ed. P. de Mayo (Academic Press, New York, 1980).

[8] B. Bagchi and V. Ramamurthy, to be published.

[9] B. Bagchi, G.R. Fleming and D.W. Oxtoby, J. Chem. Phys, 78 (1983) 7375.

[10] B. Bagchi, S. Singer and D.W. Oxtoby, Chem. Phys. Letters 99 (1983) 225.

[11] B. Bagchi, Chem. Phys. Letters 115 (1985) 209.

[12] B. Bagchi, Intern. Rev. Phys. Chem. (1986), to be published.

[13] S. Chandrasekhar, Rev. Mod. Phys. 15 (1943) 1.

[14] A. Ben-Shaul, Y. Haas, K.L. Kompa and R.D. Levine, in: Lasers and chemical change (Springer, Berlin, 1981). 